\title{
ON INTEGER SEQUENCES GENERATED BY LINEAR MAPS
}

\author{
ARTŪRAS DUBICKAS \\ Department of Mathematics and Informatics, Vilnius University, Naugarduko 24, \\ Vilnius LT-03225, Lithuania \\ E-mail: arturas.dubickas@mif.vu.lt
}

(Received 29 April 2008; accepted 9 September 2008)

\begin{abstract}
Let $x_{0}<x_{1}<x_{2}<\cdots$ be an increasing sequence of positive integers given by the formula $x_{n}=\left\lfloor\beta x_{n-1}+\gamma\right\rfloor$ for $n=1,2,3, \ldots$, where $\beta>1$ and $\gamma$ are real numbers and $x_{0}$ is a positive integer. We describe the conditions on integers $b_{d}, \ldots, b_{0}$, not all zero, and on a real number $\beta>1$ under which the sequence of integers $w_{n}=$ $b_{d} x_{n+d}+\cdots+b_{0} x_{n}, n=0,1,2, \ldots$, is bounded by a constant independent of $n$. The conditions under which this sequence can be ultimately periodic are also described. Finally, we prove a lower bound on the complexity function of the sequence $q x_{n+1}-$ $p x_{n} \in\{0,1, \ldots, q-1\}, n=0,1,2, \ldots$, where $x_{0}$ is a positive integer, $p>q>1$ are coprime integers and $x_{n}=\left\lceil p x_{n-1} / q\right\rceil$ for $n=1,2,3, \ldots$ A similar speculative result concerning the complexity of the sequence of alternatives $(F: x \mapsto x / 2$ or $S: x \mapsto(3 x+$ 1)/2) in the $3 x+1$ problem is also given.
\end{abstract}

2000 Mathematics Subject Classification. 11B50, 11B83, 11R06, 68R15.

1. Introduction. For a given real number $y$, let $\{y\},\lfloor y\rfloor$ and $\lceil y\rceil$ be the fractional part, the integral part and the ceiling function of $y$, respectively. For any real numbers $y$ and $\beta>1$, one can study the sequence of so-called $\beta$-transformations, given by $y_{0}=y$ and $y_{n}=\left\{\beta y_{n-1}\right\}$ for $n=1,2,3, \ldots$. This sequence was first investigated by Rényi [18] and Parry [17]. In particular, the sequence $y_{0}=1, y_{n}=\left\{\beta y_{n-1}\right\}$ for $n=1,2,3, \ldots$ is called the Rényi development of unity.

In fact, $y \in[0,1)$ can be expressed as

$$
y=\sum_{k=1}^{\infty} \varepsilon_{k}(y) \beta^{-k},
$$

where $\varepsilon_{k}(y)=\left\lfloor\beta y_{k-1}\right\rfloor \in\{0,1, \ldots,\lfloor\beta\rfloor\}$. This expression is called the $\beta$-expansion of $y$. In general, if $y=\sum_{k=1}^{\infty} \varepsilon_{k} \beta^{-k}$ with some $\varepsilon_{1}, \varepsilon_{2}, \varepsilon_{3}, \ldots \in\{0,1, \ldots,\lfloor\beta\rfloor\}$ then this is not necessarily the $\beta$-expansion of $y$ (see $[12,17]$ ). Clearly, the $\beta$-expansion of $y$ is ultimately periodic if and only if the sequence $y_{n}, n=0,1,2, \ldots$, is ultimately periodic. Schmidt [20] showed that if the $\beta$-expansion of every number $y \in \mathbb{Q} \cap[0,1)$ is ultimately periodic then $\beta>1$ must be either a Pisot number or a Salem number. Recall that $\beta>1$ is a Pisot number (resp. Salem number) if it is an algebraic integer whose conjugates over $\mathbb{Q}$ (if any) all lie in the open unit disc $|z|<1$ (resp. closed unit disc $|z| \leqslant 1$ with at least one conjugate lying on the circle $|z|=1$ ). Finite $\beta$-expansions have been studied in [9] and [11]; those results are also related to Pisot numbers.

In this paper, in contrast to the fractional $\beta$-transformations, we shall study a kind of integral $\beta$-transformations. Let $x_{0}$ be a positive integer, and let $\beta>1$ and $\gamma$ be two 
real numbers such that $(\beta-1) x_{0} \geqslant 1-\gamma$. Consider an increasing sequence of positive integers $x_{0}<x_{1}<x_{2}<\cdots$ generated by the map

$$
T_{\beta, \gamma}: x \mapsto\lfloor\beta x+\gamma\rfloor,
$$

namely,

$$
x_{n}=\left\lfloor\beta x_{n-1}+\gamma\right\rfloor=T_{\beta, \gamma}^{n}\left(x_{0}\right)
$$

for each $n \geqslant 1$. Indeed, $x_{n}=\left\lfloor\beta x_{n-1}+\gamma\right\rfloor>\beta x_{n-1}+\gamma-1 \geqslant x_{n-1}$ for $n \geqslant 1$, because $(\beta-1) x_{n-1} \geqslant(\beta-1) x_{0} \geqslant 1-\gamma$. For this sequence $x_{n}, n=0,1,2, \ldots$, we prove the following:

THEOREM 1. Let $b_{d}, \ldots, b_{1}, b_{0}$ be some integers, not all zero. Then the sequence

$$
w_{n}=b_{d} x_{n+d}+\cdots+b_{1} x_{n+1}+b_{0} x_{n}, \quad n=0,1,2, \ldots,
$$

is bounded by an absolute constant $B=B\left(b_{0}, \ldots, b_{d}, \beta, \gamma, x_{0}\right)$ independent of $n$ if and only if $\beta>1$ is an algebraic number and the polynomial $b_{d} X^{d}+\cdots+b_{1} X+b_{0}$ is divisible by the minimal polynomial of $\beta$ in $\mathbb{Z}[X]$.

For the sequence $x_{0}<x_{1}<x_{2}<\cdots$, where $x_{n}=\left\lfloor\beta x_{n-1}+\gamma\right\rfloor$ with an algebraic number $\beta>1$, we prove the following:

THEOREM 2. Let $\beta>1$ be an algebraic number with minimal polynomial $b_{d} X^{d}$ $+\cdots+b_{1} X+b_{0} \in \mathbb{Z}[X]$. If the sequence

$$
w_{n}=b_{d} x_{n+d}+\cdots+b_{1} x_{n+1}+b_{0} x_{n}, \quad n=0,1,2, \ldots,
$$

is ultimately periodic, then $\beta$ must be either a Pisot number or a Salem number.

Note that the conclusion of Theorem 2 is the same as that of Schmidt [20] and as that of the author [5], where $x_{n}$ was defined as $x_{n}=\left\lfloor\xi \beta^{n}\right\rfloor$ with $\xi \neq 0$. We remark that the same statements as those of Theorems 1 and 2 hold if we replace the map $T_{\beta, \gamma}: x \mapsto\lfloor\beta x+\gamma\rfloor$ by the map

$$
U_{\beta, \gamma}: x \mapsto\lceil\beta x+\gamma\rceil,
$$

where $(\beta-1) x_{0}+\gamma>0$. (See the proofs of these two theorems in Sections 2 and 3.) The condition $(\beta-1) x_{0}+\gamma>0$ implies that the sequence $x_{n}=\left\lceil\beta x_{n-1}+\gamma\right\rceil=$ $U_{\beta, \gamma}^{n}\left(x_{0}\right)$ is strictly increasing, i.e. $x_{0}<x_{1}<x_{2}<\cdots$. This sequence with $\gamma=0$ was considered by Odłyzko and Wilf [16]. They proved that if $\beta \geqslant 2$ or $\beta=2-1 / q$ with some integer $q \geqslant 2$ then $x_{n}=\left\lfloor c(\beta) \beta^{n}\right\rfloor$ for each $n \geqslant 0$ and some constant $c(\beta)$.

Clearly, if $\beta>1$ is a rational integer then $w_{n}=x_{n+1}-\beta x_{n}=0$, so the sequence considered in Theorem 2 is purely periodic. In Section 6 we shall consider the sequence $x_{0} \in \mathbb{N}, x_{n}=\left\lceil\beta x_{n-1}\right\rceil, n=1,2,3, \ldots$, with a quadratic Pisot number $\beta$. We will show that in this case the sequence $w_{n}, n=0,1,2, \ldots$, considered in Theorem 2 is also purely periodic.

Finally, let $\beta$ be a rational number which is not an integer, i.e. $\beta=p / q$, where $p>q>1$ are some coprime integers. Consider the map

$$
U_{p / q}: x \mapsto\lceil p x / q\rceil .
$$


The sequence of iterations

$$
x_{n}=\left\lceil p x_{n-1} / q\right\rceil=U_{p / q}^{n}\left(x_{0}\right), \quad n=0,1,2, \ldots,
$$

where $x_{0}$ is a positive integer, is strictly increasing $x_{0}<x_{1}<x_{2}<\cdots$. We have

$$
w_{n}=q x_{n+1}-p x_{n}=q\left\lceil p x_{n} / q\right\rceil-p x_{n} \in\{0,1, \ldots, q-1\},
$$

so the sequence $w_{n}, n=0,1,2, \ldots$, is bounded. Since $\beta=p / q$ is neither a Pisot number nor a Salem number, by Theorem 2 (see also the remark above concerning its application to $U_{\beta, \gamma}$ ), this sequence is not ultimately periodic. In case $p<q^{2}$, we can prove much more than that.

THEOREM 3. Let $w=w_{0}, w_{1}, w_{2}, \ldots$ be a sequence given by $w_{n}=q x_{n+1}-p x_{n}$, $n=0,1,2, \ldots$, where $p>q>1$ are two coprime integers, $x_{0}$ is a positive integer and $x_{n}=\left\lceil p x_{n-1} / q\right\rceil$ for each $n \geqslant 1$. Then $\liminf _{n \rightarrow \infty} P(w, n) / n \geqslant \log q / \log (p / q)$.

Here, $P(w, n)$ is the complexity function (or block-complexity function) of the sequence $w=w_{0}, w_{1}, w_{2}, \ldots$, which, for every positive integer $n$, is defined as the number of distinct vectors $\left(w_{j}, w_{j+1}, \ldots, w_{j+n-1}\right)$ of length $n$, where $j$ runs through all non-negative integers $0,1,2, \ldots$ Clearly, the function $P(w, n)$ is non-decreasing in $n$. It is bounded from above by an absolute constant independent of $n$ if and only if the sequence $w$ is ultimately periodic; otherwise, $P(w, n) \geqslant n+1$ for each positive integer $n$ (see [14] or [15]). The sequences $w$ for which equality $P(w, n)=n+1$ holds for each positive integer $n$ are called Sturmian sequences (see $[3, \mathbf{4}, \mathbf{1 4}])$. They have the lowest possible complexity among all sequences which are not ultimately periodic.

Note that in case $p<q^{2}$ the constant $\log q / \log (p / q)$ is greater than 1 . So, by Theorem $3, \liminf _{n \rightarrow \infty} P(w, n) / n>1$. In particular, this implies that the sequence $w$ considered in Theorem 3 is not Sturmian. If $p<q^{3 / 2}$ then $\log q / \log (p / q)>2$, so the sequence $w$ cannot belong to the class of Arnoux-Rauzy sequences which have complexity $2 n+1$. Since $w_{n}(\bmod q)=-p x_{n}(\bmod q)$ and $\operatorname{gcd}(p, q)=1$, the complexity $P(w, n)$ of $w$ is equal to the complexity $P(\mathcal{X}, n)$ of the sequence $\mathcal{X}=$ $x_{n}(\bmod q), n=0,1,2, \ldots$.

For $p / q=3 / 2$, the map $U_{3 / 2}$ is given by

$$
U_{3 / 2}(x)= \begin{cases}3 x / 2, & \text { if } x \text { is even } \\ (3 x+1) / 2, & \text { if } x \text { is odd }\end{cases}
$$

This map was studied in [8, p. 127]. It is related to the distribution of the fractional parts $\left\{\xi(3 / 2)^{n}\right\}, n=0,1,2, \ldots$ The sequence given by $x_{0}=1$ and $x_{n}=\left\lceil 3 x_{n-1} / 2\right\rceil=$ $U_{3 / 2}^{n}\left(x_{0}\right)$ for $n \geqslant 1$ is exactly the sequence A061419 of [21]. See also [2], where similar sequences are used for expansions of integers in rational non-integer base. A corresponding $w_{n}=2 x_{n+1}-3 x_{n}=2 U_{3 / 2}\left(x_{n}\right)-3 x_{n}$ is equal to 0 if $x_{n}$ is even, and to 1 if $x_{n}$ is odd. So $w_{n}=x_{n}(\bmod 2)$. Theorem 3 implies the following:

COROLlary 4. Let $0<x_{0}<x_{1}<x_{2}<\cdots$ be a sequence of integers given by $x_{n}=\left\lceil 3 x_{n-1} / 2\right\rceil, n=1,2,3, \ldots$ Set $X_{n}=x_{n}(\bmod 2) \in\{0,1\}$ for $n \geqslant 0$, and let $\mathcal{X}=$ $X_{0}, X_{1}, X_{2}, \ldots$ Then $P(\mathcal{X}, n)>1.70951129 n$ for each sufficiently large $n$.

This corollary is the first result which claims something more than just nonperiodicity of the sequence of iterations given by the map $U_{3 / 2}$. The famous unsolved 
$3 x+1$ problem asserts that the sequence of iterations given by a very similar map

$$
U(x)= \begin{cases}x / 2, & \text { if } x \text { is even, } \\ (3 x+1) / 2, & \text { if } x \text { is odd }\end{cases}
$$

which starts at a positive integer must end up with the cycle $2 \mapsto 1 \mapsto 2 \mapsto 1 \mapsto \cdots$. Let us write the letter $F$ for the first alternative $x \mapsto x / 2$ and the letter $S$ for the second alternative $x \mapsto(3 x+1) / 2$. Starting from 15 , we have

$$
15 \mapsto 23 \mapsto 35 \mapsto 53 \mapsto 80 \mapsto 40 \mapsto 20 \mapsto 10 \mapsto 5 \mapsto 8 \mapsto 4 \mapsto 2 \mapsto 1 \mapsto 2 \mapsto 1 \cdots .
$$

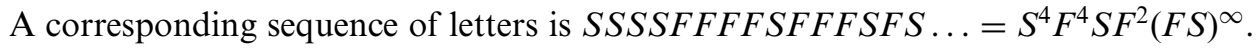
Of course, the sequence of $F, S$ is the following sequence of 0,1

$$
x_{0}, x_{1}, x_{2}, x_{3}, \ldots(\bmod 2),
$$

where $F$ corresponds to 0 and $S$ corresponds to 1 . Assume that the $3 x+1$-conjecture is false. Then there is either a non-trivial cycle or the sequence $x_{n}, n=1,2,3, \ldots$, is unbounded. In the latter case (sometimes this is called the case of divergent trajectories), we shall prove the following speculative result:

THEOREM 5. Let $x_{0}, x_{1}, x_{2}, \ldots$ be a sequence of positive integers given by $x_{n}=$ $U\left(x_{n-1}\right), n=1,2,3, \ldots$ Assume that $x_{n} \rightarrow \infty$ as $n \rightarrow \infty$. Set $X_{n}=x_{n}(\bmod 2) \in$ $\{0,1\}$ for $n \geqslant 0$, and let $\mathcal{X}=X_{0}, X_{1}, X_{2}, \ldots$ Then $P(\mathcal{X}, n)>1.70951129 n$ for each sufficiently large $n$.

For $\mathcal{X}$ given in Corollary 4, we conjecture that $P(\mathcal{X}, n)=2^{n}$ for every positive integer $n$. More generally, we conjecture that $P(w, n)=q^{n}$ for every sequence $w$ considered in Theorem 3. (See also [16], where an even stronger statement is conjectured in case $q=p-1$.) It seems very likely that this conjecture is as difficult as a corresponding conjecture claiming that the complexity function $P(\alpha, n)$ of the expansion of an algebraic irrational number $\alpha$ in base $q \geqslant 2$, i.e.

$$
\alpha=\lfloor\alpha\rfloor+\sum_{k=1}^{\infty} g_{k}(\alpha) q^{-k},
$$

$g_{k}(\alpha) \in\{0,1, \ldots, q-1\}$, defined as the complexity of the sequence $g_{k}(\alpha), k=$ $1,2,3, \ldots$, is equal to $q^{n}$. So far the equality $P(\alpha, n)=q^{n}$ is out of reach. By a result of Adamczewski and Bugeaud [1], we know that $P(\alpha, n) / n \rightarrow \infty$ as $n \rightarrow \infty$ for each algebraic irrational number $\alpha$. One among earlier results [7] implies that $P(\alpha, n)-n \rightarrow \infty$ as $n \rightarrow \infty$. Analogously, in our problem, Theorem 3 implies that $P(w, n)-n \rightarrow \infty$ as $n \rightarrow \infty$ in case $p<q^{2}$.

The sequence considered in Corollary 4 is related to the so-called Josephus problem (see, e.g., $[13,16,19])$. There are $N$ places arranged around a circle and numbered clockwise $1,2, \ldots, N$. Each of $N$ people takes one of the places. Then the $p$ th is executed. If some place is just vacated, then the $p$ th one of the remaining survivors clockwise will be executed next and so on, until just one remains. Which is the initial place $J_{p}(N)$ of the last survivor? The answer is given in terms of one of the above sequences. Given integer $p \geqslant 2$, consider the sequence $x_{0}, x_{1}, x_{2}, \ldots$ defined by $x_{0}=1$ and $x_{n}=\left\lceil p x_{n-1} /(p-1)\right\rceil$ for $n \geqslant 1$. Then

$$
J_{p}(N)=p N+1-x_{k},
$$


where $k$ is the least integer such that $x_{k}>(p-1) N$ (see, e.g., Section 3.3 in [10] or [16]).

Note that $w_{n}=(p-1) x_{n+1}-p x_{n}$ modulo $p-1$ is equal to $x_{n}(\bmod (p-1))$. Put $X_{n}=x_{n}(\bmod (p-1)) \in\{0,1, \ldots, p-2\}$. The constant

$$
K(p)=1+\frac{1}{p} \sum_{k=0}^{\infty} X_{k}\left(\frac{p-1}{p}\right)^{k}
$$

appears in [16], where the exact formula for $J_{3}(n)$ was obtained. In particular, $K(2)=1$, $K(3)=1.6222705028 \ldots$ Theorem 3 implies that, for every integer $p \geqslant 3$ and every $\varepsilon>0$, the complexity function $P(\mathcal{X}, n)$ of the sequence $\mathcal{X}=X_{0}, X_{1}, X_{2}, \ldots$ is at least $(1 /(\log p / \log (p-1)-1)-\varepsilon) n$ for each sufficiently large $n$.

2. Proof of Theorem 1. Write $x_{n+m}=\left\lfloor\beta x_{n+m-1}+\gamma\right\rfloor=\beta x_{n+m-1}+\tau_{n+m-1}$ for each $n \geqslant 0$ and each $m \geqslant 1$, where $\tau_{0}, \tau_{1}, \tau_{2}, \ldots \in(\gamma-1, \gamma]$. Then

$$
x_{n+m}=\beta^{m} x_{n}+\beta^{m-1} \tau_{n}+\beta^{m-2} \tau_{n+1}+\cdots+\tau_{n+m-1} .
$$

Applying this formula to $m=1,2, \ldots, d$ and putting corresponding values into

$$
w_{n}=b_{d} x_{n+d}+\cdots+b_{1} x_{n+1}+b_{0} x_{n},
$$

we find that

$$
w_{n}=\left(b_{d} \beta^{d}+\cdots+b_{1} \beta+b_{0}\right) x_{n}+\sum_{j=0}^{d-1} \tau_{n+j} \sum_{i=0}^{d-j-1} b_{i+j+1} \beta^{i} .
$$

Since $\left|\sum_{i=0}^{d-j-1} b_{i+j+1} \beta^{i}\right| \leqslant \beta^{d-1}\left(\left|b_{d}\right|+\cdots+\left|b_{1}\right|\right)$ and $\left|\tau_{n+j}\right|<|\gamma|+1$, the modulus of the double sum is bounded from above by $B_{0}=d \beta^{d-1}\left(\left|b_{d}\right|+\cdots+\left|b_{1}\right|\right)(|\gamma|+$ 1). Hence the sequence $w_{n}, n=0,1,2, \ldots$, is bounded by a constant $B$ independent of $n$ if and only if the term $\left(b_{d} \beta^{d}+\cdots+b_{1} \beta+b_{0}\right) x_{n}, n=0,1,2, \ldots$, is bounded. However, $x_{n} \rightarrow \infty$ as $n \rightarrow \infty$, because $x_{0}<x_{1}<x_{2}<\cdots$ is strictly increasing. Evidently, if there is a constant $B_{1}$ independent of $n$ such that $\left|\left(b_{d} \beta^{d}+\cdots+b_{1} \beta+b_{0}\right) x_{n}\right| \leqslant B_{1}$ for each $n \geqslant 0$ then $b_{d} \beta^{d}+\cdots+b_{1} \beta+b_{0}=0$. Hence $b_{d} X^{d}+\cdots+b_{1} X+b_{0}$ is divisible by the minimal polynomial of $\beta$ in $\mathbb{Z}[X]$.

On the other hand, if $b_{d} X^{d}+\cdots+b_{1} X+b_{0}$ is divisible by the minimal polynomial of an algebraic number $\beta$ then $b_{d} \beta^{d}+\cdots+b_{1} \beta+b_{0}=0$. Thus $\left|w_{n}\right|=$ $\left|\sum_{j=0}^{d-1} \tau_{n+j} \sum_{i=0}^{d-j-1} b_{i+j+1} \beta^{i}\right|$ is bounded from above by the constant $B_{0}=d \beta^{d-1}\left(\left|b_{d}\right|+\right.$ $\left.\cdots+\left|b_{1}\right|\right)(|\gamma|+1)$.

3. Proof of Theorem 2. Below, we shall use the following lemma (which is a special case of Lemma 1 in [6]):

LEMMA 6. Let $a_{d} X^{d}+\cdots+a_{1} X+a_{0}=a_{d}\left(X-\alpha_{1}\right) \cdots\left(X-\alpha_{d}\right) \in \mathbb{Z}[X]$ be an irreducible polynomial, $a_{d}>0$, and let $z_{n}, n=0,1,2, \ldots$ be a sequence of integers satisfying $a_{d} z_{n+d}+\cdots+a_{1} z_{n+1}+a_{0} z_{n}=0$ for each $n \geqslant n_{0}$. Then $\alpha=\alpha_{1}$ is an algebraic integer, namely $a_{d}=1$, and there is a polynomial $Q(X) \in \mathbb{Q}[X]$ such that

$$
z_{n}=Q\left(\alpha_{1}\right) \alpha_{1}^{n}+\cdots+Q\left(\alpha_{d}\right) \alpha_{d}^{n}
$$


for each $n \geqslant n_{0}$.

Suppose that there is a positive integer $t$ such that $w_{n}=w_{n+t}$ for each $n \geqslant n_{0}$. Then

$$
b_{d}\left(x_{n+d+t}-x_{n+d}\right)+\cdots+b_{1}\left(x_{n+1+t}-x_{n+1}\right)+b_{0}\left(x_{n+t}-x_{n}\right)=0
$$

for each $n \geqslant n_{0}$. Here, each difference $x_{n+j+t}-x_{n+j}$, where $j=0,1, \ldots, d$, is a positive integer. Hence, by Lemma 6 , there exists a polynomial $G(X)$ with rational coefficients such that

$$
x_{n+t}-x_{n}=G\left(\beta_{1}\right) \beta_{1}^{n}+\cdots+G\left(\beta_{d}\right) \beta_{d}^{n}
$$

for each $n \geqslant n_{0}$, where $\beta_{1}=\beta, \beta_{2}, \ldots, \beta_{d}$ are the conjugates of $\beta>1$ over $\mathbb{Q}$, and $\beta$ must be an algebraic integer, i.e. $b_{d}=1$. If $d=1$, namely, $\beta>1$ is a rational number, then $\beta$ must be a positive integer greater than 1 . So it is a Pisot number.

Suppose that $d \geqslant 2$. Using the inequality

$$
\left|x_{n+1}-\beta x_{n}\right|=\left|\left\lfloor\beta x_{n}+\gamma\right\rfloor-\beta x_{n}\right|=\left|\gamma-\left\{\beta x_{n}+\gamma\right\}\right|<|\gamma|+1,
$$

which holds for each $n \geqslant 0$, we deduce that

$$
\left|\sum_{j=1}^{d} G\left(\beta_{j}\right) \beta_{j}^{n+1}-\beta_{1} \sum_{j=1}^{d} G\left(\beta_{j}\right) \beta_{j}^{n}\right|=\left|x_{n+t+1}-x_{n+1}-\beta\left(x_{n+t}-x_{n}\right)\right|<2(|\gamma|+1) .
$$

So the modulus of

$$
\delta_{n}=\sum_{j=1}^{d} G\left(\beta_{j}\right) \beta_{j}^{n+1}-\beta_{1} \sum_{j=1}^{d} G\left(\beta_{j}\right) \beta_{j}^{n}=\sum_{j=2}^{d}\left(\beta_{j}-\beta_{1}\right) G\left(\beta_{j}\right) \beta_{j}^{n}
$$

is smaller than $2(|\gamma|+1)$ for every $n \geqslant n_{0}$.

Taking $d-1$ consecutive equations for $\delta_{n}, \ldots, \delta_{n+d-2}$, where $n \geqslant n_{0}$ and

$$
\delta_{n+i}=\sum_{j=2}^{d}\left(\beta_{j}-\beta_{1}\right) \beta_{j}^{i} G\left(\beta_{j}\right) \beta_{j}^{n}, \quad i=0,1, \ldots, d-2,
$$

we see that the vector $\left(\beta_{2}^{n}, \ldots, \beta_{d}^{n}\right)$ is a solution of a non-homogeneous linear system. By Cramer's rule, this linear system has a unique solution, because the corresponding matrix $A=\left(\left(\beta_{j}-\beta_{1}\right) G\left(\beta_{j}\right) \beta_{j}^{i}\right)_{0 \leqslant i \leqslant d-2,2 \leqslant j \leqslant d}$ is non-singular. Indeed, its determinant is equal to the Vandermonde determinant $\prod_{2 \leqslant k<j \leqslant d}\left(\beta_{j}-\beta_{k}\right)$ multiplied by the factor $\prod_{j=2}^{d}\left(\beta_{j}-\beta_{1}\right) G\left(\beta_{j}\right)$. Here $\left(\beta_{j}-\beta_{1}\right) G\left(\beta_{j}\right) \neq 0$ for $j=2, \ldots, d$, because $G\left(\beta_{j}\right) \neq 0$ for each $j$. Hence the matrix $A$ is non-singular.

Now, using the fact that $\left|\delta_{n}\right|, \ldots,\left|\delta_{n+d-2}\right|<2(|\gamma|+1)$, by Cramer's rule, we deduce that each $\left|\beta_{j}^{n}\right|$, where $j=2, \ldots, d$ and $n \geqslant n_{0}$, is bounded from above by a constant $C$ independent of $n$. The inequality $\left|\beta_{j}^{n}\right| \leqslant C$, where $n=n_{0}, n_{0}+1, n_{0}+$ $2, \ldots$, shows that $\left|\beta_{j}\right| \leqslant 1$. Thus $\left|\beta_{j}\right| \leqslant 1$ for every $j=2, \ldots, d$. Since $\beta=\beta_{1}>1$ is an algebraic integer, we conclude that $\beta$ must be either a Pisot number or a Salem number. 
4. Proof of Theorem 3. Since $w_{n}=q x_{n+1}-p x_{n} \in\{0,1, \ldots, q-1\}$ for each $n \geqslant$ 0 , expressing $x_{n+m}$ as $p x_{n+m-1} / q+w_{n+m-1} / q$ and so on, we obtain

$$
x_{n+m}=(p / q)^{m} x_{n}+q^{-1}\left((p / q)^{m-1} w_{n}+(p / q)^{m-2} w_{n+1}+\cdots+w_{n+m-1}\right) .
$$

Suppose that the $\operatorname{limit} \lim \inf _{n \rightarrow \infty} P(w, n) / n$ is strictly smaller than $\log q / \log (p / q)$. Then there is an infinite sequence of positive integers $m_{1}<m_{2}<m_{3}<\cdots$ such that $P\left(w, m_{k}\right) \leqslant m_{k}(\log q / \log (p / q)-\varepsilon)$ for some $\varepsilon>0$ and each $k \geqslant 1$.

Set $m=m_{k}$ for some fixed $k \geqslant 1$ which is so large that

$$
\varepsilon m_{k} \log (p / q)>\log \left(x_{0}+q-1\right) .
$$

Consider the vectors $\left(w_{n}, w_{n+1}, \ldots, w_{n+m-1}\right)$ for $n=0,1, \ldots,\lfloor m(\log q / \log (p / q)-\varepsilon)\rfloor$. There are more than $m(\log q / \log (p / q)-\varepsilon) \geqslant P(w, m)$ of such vectors, so at least two of them must be equal, say $\left(w_{s}, \ldots, w_{s+m-1}\right)=\left(w_{n}, \ldots, w_{n+m-1}\right)$, where $0 \leqslant s<n \leqslant$ $\lfloor m(\log q / \log (p / q)-\varepsilon)\rfloor$. Subtracting

$$
x_{s+m}=(p / q)^{m} x_{s}+q^{-1}\left((p / q)^{m-1} w_{s}+(p / q)^{m-2} w_{s+1}+\cdots+w_{s+m-1}\right)
$$

from $x_{n+m}$, we deduce that

$$
x_{n+m}-x_{s+m}=(p / q)^{m}\left(x_{n}-x_{s}\right) .
$$

Hence $q^{m}$ divides $x_{n}-x_{s}$. Since $x_{n}>x_{s}>0$, this implies that $q^{m}$ must be smaller than $x_{n}$. But

$$
x_{n}=(p / q)^{n} x_{0}+q^{-1}\left((p / q)^{n-1} w_{0}+(p / q)^{n-2} w_{1}+\cdots+w_{n-1}\right),
$$

so, using $w_{j} \leqslant q-1$, we deduce that

$$
q^{m}<x_{n} \leqslant(p / q)^{n} x_{0}+\left((p / q)^{n}-1\right)(q-1) /(p-q)<(p / q)^{n}\left(x_{0}+q-1\right) .
$$

By taking the logarithms of both sides and using

$$
n \leqslant\lfloor m(\log q / \log (p / q)-\varepsilon)\rfloor \leqslant m(\log q / \log (p / q)-\varepsilon),
$$

we obtain

$$
\begin{aligned}
m \log q< & \log x_{n}<n \log (p / q)+\log \left(x_{0}+q-1\right) \leqslant m \log q-\varepsilon m \log (p / q) \\
& +\log \left(x_{0}+q-1\right) .
\end{aligned}
$$

It follows that $\varepsilon m_{k} \log (p / q)=\varepsilon m \log (p / q)<\log \left(x_{0}+q-1\right)$, contrary to our assumption on $m_{k}$.

5. Proof of Theorem 5. Note that $x_{n+1}=\left(u_{n} x_{n}+v_{n}\right) / 2$, where $\left(u_{n}, v_{n}\right)=(1,0)$ if $X_{n}=x_{n}(\bmod 2)=0$ and $\left(u_{n}, v_{n}\right)=(3,1)$ if $X_{n}=x_{n}(\bmod 2)=1$. Let $n \geqslant 0$ and $m \geqslant 1$ be two integers. Expressing $x_{n+m}$ by $x_{n+m-1}$ and so on up to $x_{n}$, we obtain

$$
x_{n+m}=\frac{u_{n+m-1} \cdots u_{n} x_{n}}{2^{m}}+\frac{u_{n+m-1} \cdots u_{n+1} v_{n}}{2^{m}}+\frac{u_{n+m-1} \cdots u_{n+2} v_{n+1}}{2^{m-1}}+\cdots+\frac{v_{n+m-1}}{2} \text {. }
$$


Suppose that the limit $\lim _{\inf _{n \rightarrow \infty}} P(\mathcal{X}, n) / n$ is strictly smaller than $\log 2 / \log (3 / 2)$. Then there is an infinite sequence of positive integers $m_{1}<m_{2}<m_{3}<\cdots$ such that $P\left(\mathcal{X}, m_{k}\right) \leqslant m_{k}(\log 2 / \log (3 / 2)-\varepsilon)$ for some $\varepsilon>0$ and each $k \geqslant 1$.

Fix any $m \in\left\{m_{1}, m_{2}, m_{3}, \cdots\right\}$ satisfying

$$
m \log (3 / 2)>\varepsilon^{-1} \log \left(x_{0}+1\right) .
$$

Consider the vectors $\left(X_{n}, X_{n+1}, \ldots, X_{n+m-1}\right)$ for $n=0,1, \ldots,\lfloor m(\log 2 / \log (3 / 2)-\varepsilon)\rfloor$. There are more than $m(\log 2 / \log (3 / 2)-\varepsilon) \geqslant P(\mathcal{X}, m)$ of such vectors. Hence, at least two of them must be equal, for instance $\left(X_{s}, \ldots, X_{s+m-1}\right)=\left(X_{n}, \ldots, X_{n+m-1}\right)$, where $0 \leqslant s<n \leqslant\lfloor m(\log 2 / \log (3 / 2)-\varepsilon)\rfloor$. Subtracting

$$
x_{s+m}=\frac{u_{s+m-1} \cdots u_{s} x_{s}}{2^{m}}+\frac{u_{s+m-1} \cdots u_{s+1} v_{s}}{2^{m}}+\frac{u_{s+m-1} \cdots u_{s+2} v_{s+1}}{2^{m-1}}+\cdots+\frac{v_{s+m-1}}{2}
$$

from a corresponding expression for $x_{n+m}$ and using $u_{n+j}=u_{s+j}, v_{n+j}=v_{s+j}$ for $j=$ $0,1, \ldots, m-1$, we derive that

$$
x_{n+m}-x_{s+m}=\frac{u_{n+m-1} \cdots u_{n}}{2^{m}}\left(x_{n}-x_{s}\right) .
$$

Recall that $u_{k} \in\{1,3\}$, so $\operatorname{gcd}\left(u_{n+m-1} \cdots u_{n}, 2^{m}\right)=1$. Hence $2^{m}$ divides $\left|x_{n}-x_{s}\right|$. We claim that $x_{n} \neq x_{s}$. Indeed, if $x_{n}=x_{s}$ then the sequence $x_{s}, x_{s+1}, x_{s+2}, \ldots$ is an infinite repetition of the string $x_{s}, \ldots, x_{n-1}$. So the sequence $x_{0}, x_{1}, x_{2}, \ldots$ is bounded, contrary to the condition of the theorem. From

$$
x_{n}=\frac{u_{n-1} \cdots u_{0} x_{0}}{2^{n}}+\frac{u_{n-1} \cdots u_{1} v_{0}}{2^{n}}+\frac{u_{n-1} \cdots u_{2} v_{1}}{2^{n-1}}+\cdots+\frac{v_{n-1}}{2},
$$

using $u_{k} \in\{1,3\}, \quad v_{k} \in\{0,1\}$, we derive that $x_{n}<(3 / 2)^{n}\left(x_{0}+1\right)$. Similarly, $x_{s}<$ $(3 / 2)^{s}\left(x_{0}+1\right)$. Hence,

$$
2^{m} \leqslant\left|x_{n}-x_{s}\right|<(3 / 2)^{n}\left(x_{0}+1\right),
$$

because $n>s$.

By taking the logarithms and using

$$
n \leqslant\lfloor m(\log 2 / \log (3 / 2)-\varepsilon)\rfloor \leqslant m(\log 2 / \log (3 / 2)-\varepsilon),
$$

we obtain

$$
m \log 2<n \log (3 / 2)+\log \left(x_{0}+1\right) \leqslant m \log 2-\varepsilon m \log (3 / 2)+\log \left(x_{0}+1\right) .
$$

Consequently, $m \log (3 / 2)<\varepsilon^{-1} \log \left(x_{0}+1\right)$, contrary to our assumption on $m$.

Therefore, $\liminf \operatorname{in}_{n \rightarrow \infty} P(\mathcal{X}, n) / n \geqslant \log 2 / \log (3 / 2)$, giving $P(\mathcal{X}, n)>1.70951129 n$ for each sufficiently large $n$.

6. Examples. Let us take $\beta=(1+\sqrt{5}) / 2$. Consider the map $x \mapsto\lceil\beta x\rceil$ and a sequence of iterations $x_{0}=1, x_{n}=\left\lceil\beta x_{n-1}\right\rceil$ associated to it. Clearly, the golden mean $(1+\sqrt{5}) / 2$ is a Pisot number, because its conjugate $\theta=(1-\sqrt{5}) / 2$ lies in $(-1,0)$. We claim that

$$
x_{n}=F_{n+2}-1 \text { for each } n \geqslant 0 .
$$


Here $F_{n}$ is the $n$th Fibonacci number, given by $F_{0}=F_{1}=1, F_{n+2}=F_{n+1}+F_{n}$.

We will show first that

$$
x_{n+2}=x_{n+1}+x_{n}+1
$$

for each $n \geqslant 0$. Indeed, writing $x_{n+1}=\beta x_{n}+\tau_{n}$ and $x_{n+2}=\beta x_{n+1}+\tau_{n+1}$, where $\tau_{n}, \tau_{n+1} \in(0,1)$, we obtain

$$
\begin{aligned}
x_{n+2}-x_{n+1}-x_{n} & =\beta x_{n+1}+\tau_{n+1}-x_{n+1}-x_{n} \\
& =\left(\beta^{2}-\beta-1\right) x_{n}+(\beta-1) \tau_{n}+\tau_{n+1}=(\beta-1) \tau_{n}+\tau_{n+1} .
\end{aligned}
$$

Since $x_{n+2}-x_{n+1}-x_{n} \in(0, \beta)$ is an integer, it is equal to 1 . Hence $x_{n+2}=x_{n+1}+x_{n}+$ 1 , as claimed. In particular, we see that, for the sequence $x_{0}, x_{1}, x_{2}, \ldots$, a corresponding sequence $w_{n}=x_{n+2}-x_{n+1}-x_{n}, n=0,1,2, \ldots$, considered in Theorem 2 is purely periodic.

Next, using $x_{0}=1=F_{2}-1$ and $x_{1}=\lceil\beta\rceil=2=F_{3}-1$, by induction on $n$, we find that

$$
x_{n+2}=x_{n+1}+x_{n}+1=F_{n+3}-1+F_{n+2}-1+1=F_{n+3}+F_{n+2}-1=F_{n+4}-1,
$$

so the formula $x_{n}=F_{n+2}-1$ holds for each $n \geqslant 0$.

More generally, let $\beta$ be a quadratic Pisot number with minimal polynomial $X^{2}-$ $a X+b$. Consider the sequence which starts with an arbitrary positive integer $x_{0}$ and is given by the formula $x_{n}=\left\lceil\beta x_{n-1}\right\rceil$ for $n \geqslant 1$. Let $\beta^{\prime}$ be the conjugate of $\beta$, i.e. $X^{2}-a X+b=(X-\beta)\left(X-\beta^{\prime}\right)$. Writing $x_{n+1}=\beta x_{n}+\tau_{n}$, we find that

$$
\begin{aligned}
w_{n} & =x_{n+2}-a x_{n+1}+b x_{n}=(\beta-a) x_{n+1}+\tau_{n+1}+b x_{n} \\
& =((\beta-a) \beta+b) x_{n}+\tau_{n+1}+(\beta-a) \tau_{n}=\tau_{n+1}+(\beta-a) \tau_{n} .
\end{aligned}
$$

Since $0<\tau_{n}, \tau_{n+1}<1$ and $\beta-a=-b / \beta=-\beta^{\prime}$, where $-1<\beta^{\prime}<1$, we see that $w_{n} \in$ $\left(0,1-\beta^{\prime}\right)$ if $\beta^{\prime}$ is negative and $w_{n} \in\left(-\beta^{\prime}, 1\right)$ if $\beta^{\prime}$ is positive. It follows that, for each $n \geqslant 0$, we have $w_{n}=1$ if $\beta^{\prime}<0$ and $w_{n}=0$ if $\beta^{\prime}>0$. In both cases, the sequence $w_{n}$, $n=0,1,2, \ldots$, is purely periodic.

\section{REFERENCES}

1. B. Adamczewski and Y. Bugeaud, On the complexity of algebraic numbers I. Expansions in integer bases, Ann. Math. 165 (2007), 547-565.

2. S. Akiyama, C. Frougny and J. Sakarovitch, Powers of rationals modulo 1 and rational base number systems, Isr. J. Math. 168 (2008), 53-91.

3. J.-P. Allouche and J. Shallit, Automatic sequences. Theory, applications, generalizations (Cambridge University Press, Cambridge, UK, 2003).

4. J. Berstel and J. Karhumäki, Combinatorics on words - a tutorial, in Current trends in theoretical computer science. The challenge of the new century, Vol. 2: Formal models and semantics (Paun, G. Rozenberg, G. and Salomaa, A., Editors), (World Scientific, River Edge, N J, 2004), 415-475.

5. A. Dubickas, Arithmetical properties of powers of algebraic numbers, Bull. Lond. Math. Soc. 38 (2006), 70-80.

6. A. Dubickas, Arithmetical properties of linear recurrent sequences, J. Number Theory 122 (2007), 142-150. 
7. S. Ferenczi and C. Mauduit, Transcendence of numbers with a low complexity expansion, J. Number Theory 67 (1997), 146-161.

8. L. Flatto, J. C. Lagarias and A. D. Pollington, On the range of fractional parts $\left\{\xi(p / q)^{n}\right\}$, Acta Arith. 70 (1995), 125-147. $713-723$

9. C. Frougny and B. Solomyak, Finite $\beta$-expansions, Ergodic Theory Dyn. Syst. 12 (1994),

10. R. L. Graham, D. E. Knuth and O. Patashnik, Concrete mathematics (Addison-Wesley, New York, 1989).

11. L. S. Guimond, Z. Masáková, and E. Pelantová, Arithmetics of beta-expansions, Acta Arith. 112 (2004), 23-40.

12. S. Ito and Y. Takahashi, Markov subshifts and the realization of $\beta$-expansions, J. Math. Soc. Jpn. 26 (1974), 33-55. 173.

13. F. Jakobczyk, On the generalized Josephus problem, Glasgow Math. J. 14 (1973), 168-

14. M. Lothaire, Algebraic combinatorics on words, Encyclopedia of mathematics and its applications, Vol. 90 (Cambridge University Press, Cambridge, UK, 2002).

15. M. Morse and G. A. Hedlund, Symbolic dynamics II: Sturmian sequences, Am. J. Math. 62 (1940), 1-42.

16. A. Odłyzko and H. Wilf, Functional iteration and the Josephus problem, Glasgow Math. J. 33 (1991), 235-240.

17. W. Parry, On the $\beta$-expansions of real numbers, Acta Math. Sci. Hung. 11 (1960), 401-416.

18. A. Rényi, Representations for real numbers and their ergodic properties, Acta Math. Sci. Hung. 8 (1957), 477-493.

19. W. J. Robinson, The Josephus problem, Math. Gaz. 44 (1960), 47-52.

20. K. Schmidt, On periodic expansions of Pisot numbers and Salem numbers, Bull. Lond. Math. Soc. 12 (1980), 269-278.

21. N. J. A. Sloane, The on-line encyclopedia of integer sequences, http://www.research. att.com $/ \sim$ njas/sequences. 